\title{
41 \\ SEARCHING WITHOUT A KEYBOARD IN A MULTIMEDIA ENVIRONMENT
}

\author{
Laura Leventhal, Barbee Teasley, Keith Instone and Daryl Stone \\ Computer-Human Interaction Laboratory \\ Computer Science Department \\ Bowling Green State University \\ Bowling Green, Ohio USA 43403 \\ Email: leventhal@cs.bgsu.edu
}

KEY WORDS: multimedia, searching, information kiosks, hypertext

\begin{abstract}
Designers of multimedia environments are challenged to develop search tools that are both appropriate and usable. The task is particularly difficult when textoriented, keyboard-based searching tools are not feasible. For example, keyboardless searching tools may be necessary for information kiosks. While prior studies have looked at keyboardless searching for small databases, the current study examines children's use of different search techniques and tools in a multimedia system. The results showed that, given well-designed tools, users can be highly effective and efficient in finding various sorts of information without using a keyboard.
\end{abstract}

\section{INTRODUCTION}

Hypertext and multimedia facilitate the presentation of graphics, text, animation, videos and sound. Designers of multimedia environments are challenged to develop search tools that are both appropriate and usable. The task is particularly difficult when text-oriented, keyboardbased searching tools are not feasible. For example, keyboardless searching tools may be necessary for information kiosks, for reasons of security and durability of the hardware, as well as for training and usability reasons. Many people who are potential users of a kiosk do not have typing skills. Young children and non-native speakers may be especially challenged by keyboard entry. Some children may be preliterate, while others may be literate, yet not have typing skills. Non-native speakers may have typing skills, but not for a particular style of language-specific keyboard. In addition, nonnative speakers may have reading fluency, but not spelling or composition fluency in a language. (Heller, 1991, delineates characteristics of kiosks and Leventhal, Instone and Teasley, 1994, discuss design issues relating to keyboardless searching in kiosks in detail.)

One kiosk system that does not rely on keyboard entry for searching is the Attendee Yearbook of the CHI '89 InfoBooth (Salomon, 1990). The yearbook is a hypertext system that contains a separate, single card (one screen) of information about each CHI ' 89 attendee in the database. In addition to the Attendee cards, there is a single Yearbook card which is the entry point into the subsystem, and Index cards, which are alphabetical lists of attendees that are sorted by first or last name. The original version of InfoBooth, used at CHI ' 89 , implemented two nonkeyboard kiosk-style search modes: linear and hierarchical (indexed). In the hierarchical mode, the user began at an Index card for a specific letter. From the Index card, the user could select a name, which would take them to the associated Attendee card. In the linear mode, the user navigated among Attendee cards. Clicking on Next and Previous buttons allowed the user to move up and down alphabetically among the Attendees. Salomon (1990) collected usability data at the conference and found that users had difficulty searching because they could not find a means of entering the hierarchical mode. Thus, they were "trapped" in the linear mode and had difficulty searching for Attendee cards that were linearly distant from their current card. In a followup study, Leventhal, Instone and Teasley (1994) created two new versions of the original InfoBooth. The format and content of the new 
versions were identical to the original. One new version encouraged a hierarchical search and a second version encouraged a linear search. Computing professionals were asked to find ten pieces of information, using one of the three versions of the system. In terms of speed and minimizing the number of cards visited, we found that the hierarchical approach was far superior to either the original version, or the linear version. This research shows that searching a relatively small database with a restricted set of possible search objectives can be quite effectively done in a non-keyboard kiosk-style entry mode, given the right tools. However, because the database of the Attendee Yearbook is relatively small (hundreds of cards), it is difficult to know whether this conclusion would scale up to a larger database.

An open question for designers of hypertext and multimedia systems is whether non-keyboard, kiosk-style searching tools can be used effectively for a large database. To this end, we performed a usability study of Dinosaur Adventure, a commercially available multimedia system (by Knowledge Adventure, Inc.) that offers a variety of keyboardless search tools. Our users were elementary school children. The children were asked to find answers to questions; the questions varied in terms of the types of tools that were most appropriately used to find the answers. We wished to determine whether the users were able to use the appropriate tools to answer the questions, and how the tool choice affected performance. More broadly, our goal was to begin delineating the parameters of effective design for non-keyboard based, kiosk-style searching.

\section{METHOD}

\subsection{Materials}

Dinosaur Adventure is a multimedia package created for "children ages 5 - 105" (Hill, 1993). It integrates sound, text, graphics, videos and animation in a kiosk interface. The main focus of the package is on different sorts of dinosaurs; however, it also includes information about the formation of the earth, the evolution of dinosaurs, and prehistoric mammals. Dinosaur Adventure is composed of five distinct activities, including a story, games and videos. The current study, however, focused on the section of this system called World of Dinosaurs. A typical screen for the World of Dinosaurs is shown in Figure 1. The screen contains four expandable windows: a picture window, a globe window, a text window, and a tool window. Each of these provides the user with one or more tools for searching. The methods of search supported by each of the window/tool combinations is described below.

Geographic search method The window in the upper left corner of the screen of World of Dinosaurs contains a globe that we called the Geographic Tool. The Geographic Tool is both an input (searching) and output device. As an input device, "hot spots" on the maps are indicated by red dots. Clicking on a hot spot activates an associated graphic (which appears in the graphics window in the lower right) and text about a dinosaur which came from the selected location (which appears in the text window on the lower left). The user rotates the globe with the four buttons located at the lower left of the globe and zooms in or out by using the slide bar at the lower right of the tool. Zooming in results in more and more detailed maps, to the level of individual countries, states and provinces. The Geographic Tool also acts as an output device in the sense that the location on the globe corresponds to whatever is the description in the text and graphics. As shown in Figure 1, the graphic and text window contain information on the oceangoing reptile, Plesiosaurus, which lived near Germany, and Germany is shown on the globe.

Index search method The upper right corner of the opening screen (Figure 1), contains a palette of tools. One of these is an icon of a library card catalog. By clicking on the card catalog icon, the user is taken directly to the Index. The Index Tool contains an alphabetical, hierarchical listing of all topics in the entire database. The first level of the hierarchy is a clickable list of the alphabet. Inside an index for a specific letter, items may be listed as either a separate topic, or as one of a group of related topics, or both. For example, "Allosaurus" is one of four terms listed under the topic "Allosaurus." "Allosaurus" is also listed under the topics "Dinosaur" and "Packs." This grouping of terms under topics imposes a second level of hierarchy to the index structure in many cases (i.e., cross-referencing). When the user selects an item from the index, the modal index window disappears and the text and graphic windows show the chosen topic.

Hypertext search method The text window in the lower left corner of the opening screen contains what might be considered traditional hypertext. The window contains text which may be scrolled using a scroll bar. In addition, clicking on certain words in the text results in a display of a related index window. In Figure 1, clicking on "marine" would activate the " $M$ " section of the 


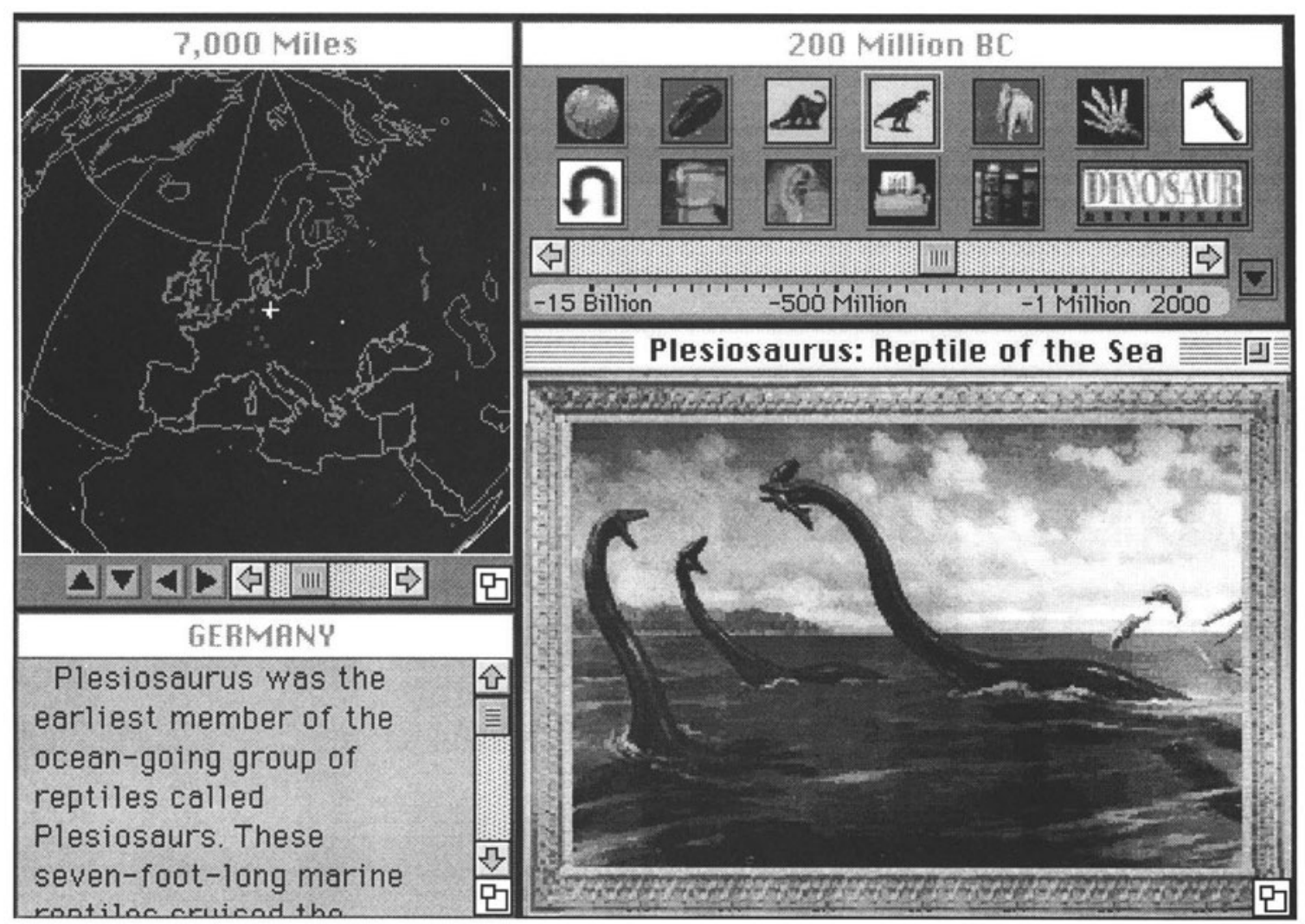

Figure 1: A Typical Screen for World of Dinosaurs

index, scrolled to the section "Marine." Users might then jump to "Elasmosaurus" since it is an entry under "Marine." Note that the clickable words are in no way visually distinguishable from the non-clickable.

Also, moving the pointer over parts of the graphic causes a text balloon containing a note of relevance to appear. Clicking on one of these areas initiates a jump to a related topic. For example, clicking on the water in the graphic of Figure 1 brings up the entry on "Water Reptiles."

Slide bar search method Another tool in the tool palette is a slide bar. The three modes of the bar are time period, height of a dinosaur, or its weight, with time period as the initial mode. To set the mode of the slide bar, the user clicks on the down arrow to the right of the slide bar. Once the mode has been set, the user can move the slide bar horizontally to select a dinosaur of an approximate relative length or size, or from an approximate period. Once the slide bar is stationary, text and a graphic describing the appropriate dinosaur appear. The information in the slide bar is also coordinated with the text and graphics windows. In Figure 1, the slide bar indicates that Plesiosaurus lived about 200 million years ago. The Slide Bar Tool also provides output in the sense that it is updated to match the text and graphics in the other windows, regardless of the search technique that located the entry.

Category search method Seven of the icons on the tool palette give direct access to seven different categories of information: Earth, Early Life, Herbivores, Carnivores, Mammals, Fossils, and History. When the user clicks on any category button, they are shown the next alphabetical entry in the database that relates to that category. For example, if the slide bar is in the time mode and is set to the year 1900, when the user clicks on the Fossils category button, the nearest entry (from the current location in the database) about fossils that is dated later than 1900 will be displayed.

\subsection{Users}

The participants were recruited from the sixth and seventh grade at St. Aloysius Elementary School 


\begin{tabular}{|c|c|c|c|c|}
\hline Question & Short name & Best Method & One/Linked & Answer Location \\
\hline What dinosaur lived in Alaska? & Alaska & Geographic & One & Text \\
\hline $\begin{array}{l}\text { What dinosaur has a strange back } \\
\text { ornament like the Dimetrodon? }\end{array}$ & Dimetrodon & Hypertext & Linked & Graphic \\
\hline $\begin{array}{l}\text { Who was the largest dinosaur by } \\
\text { height? }\end{array}$ & Height & Slide Bar & One & Text \\
\hline $\begin{array}{c}\text { Name another dinosaur that lived in } \\
\text { the same kind of place as the } \\
\text { Brachiosaurus. }\end{array}$ & Brachiosaurus & Hypertext & Linked & Text \\
\hline How many teeth did Allosaurus have? & Allosaurus & Index & One & Text \\
\hline $\begin{array}{l}\text { Give the name and describe the teeth } \\
\text { of the animal which lives in Kansas. }\end{array}$ & Kansas & Geographic & One & Graphic \\
\hline What color is Pteranodon? & Pteranodon & Index & One & Graphic \\
\hline $\begin{array}{l}\text { What is the length of the smallest } \\
\text { mammal? }\end{array}$ & Mammal & Category & One & Text \\
\hline
\end{tabular}

Table 1: Questions Used in the Dinosaur Adventure Study

in Bowling Green, Ohio, USA, and ranged in age from 10 to 12 years old. There were a total of 17 users, with 12 boys and 5 girls.

\subsection{Task and Questions}

Our goal in this study was to explore the effectiveness of different search tools, under a range of question conditions, within the limitations of our users (children's) attention span and motivation. To that end, users answered eight questions that were strategically chosen to be easily answered using a particular search technique; however all information was, in fact, eventually accessible using any of the search methods. For example, the question "What dinosaur lived in Alaska?" was most appropriately answered with the Geographic Tool. In addition, the questions varied in complexity, requiring either one fact or two, linked facts to answer. The one-fact questions (e.g., "Who was the largest dinosaur by height?") could be answered by accessing a single text entry or a single graphic. The linked questions required the user to access at least two different entries (e.g., "What dinosaur had a strange back ornament like the Dimetrodon?"), where finding one piece of information put the user a single step (link) away from the rest of the information. Finally, the questions varied in the location of the answer in the presented information. For some questions, the answer, or a part of it, was in the text and for other questions, the answer could be found in the graphic. Our previous research has demonstrated that users often have trouble extracting information from graphics in hypertext databases (Instone, Brown, Leventhal, Teasley, 1993); hence, we felt that the questions with answers in the graphics were likely more difficult than the questions with answers in the text. The entire set of questions is shown in Table 1 .

\subsection{Procedure}

Every user was trained for mouse skills using an interactive training program. The experimenter then gave an overview of Dinosaur Adventure, and demonstrated each of the five search techniques. The users were told that their job was to find the answers to eight questions and write the answers on the question sheet. They were told they could write "don't know" if they wished to go on. They were also informed that the answers might appear in either the text window or the graphic window. Users were videotaped. Two different random orders of the questions were used.

\section{RESULTS}

\subsection{Tool Use Patterns}

Our main goal of this study was to explore how well users were able to employ non-keyboard kiosk-style tools to answer questions that varied 


\begin{tabular}{|lccc|}
\hline Question & $\begin{array}{c}\text { \% of users choosing } \\
\text { best tool }\end{array}$ & $\begin{array}{c}\text { Speed } \\
\text { (seconds) }\end{array}$ & $\begin{array}{c}\text { Accuracy } \\
\text { (mean \% correct) }\end{array}$ \\
\hline Alaska & 64.7 & 171.2 & 94.1 \\
Allosaurus & 88.2 & 91.8 & 94.1 \\
Height & 70.6 & 114.7 & 52.9 \\
Mammal & 35.3 & 40.6 & 88.2 \\
Brachiosaurus & 0.0 & 224.1 & 35.3 \\
Kansas & 52.9 & 225.9 & 88.2 \\
Pteranodon & 88.2 & 155.3 & 94.1 \\
Dimetrodon & 0.0 & 187.8 & 64.7 \\
\hline
\end{tabular}

Table 2: Results for the eight questions

by appropriate tool, difficulty level and location of desired information. We recorded the number of users that selected the appropriate tool for each question and calculated overall percentages. (See Table 2 for a summary.)

For questions that were most appropriately answered with either the Index Tool (Pteranodon and Allosaurus) or the Slide Bar Tool (Height), most users used only the correct tool (more than $88 \%$ of users for the Index Tool question, $70.6 \%$ of users for the Slide Bar Tool question). Clearly users understood which tools were appropriate for these two categories of questions, regardless of question difficulty.

The two questions that were best answered with the Hypertext Tool (Dimetrodon and Brachiosaurus) were never answered with the correct tool. As an alternative, $41.2 \%$ and $58.9 \%$ of users, respectively, used the Index Tool only, while the remaining users chose a combination of tools. For the Dimetrodon question, where the link was embedded in the graphic, we observed from the videotapes that many users did not read the balloon message which appeared above the picture of the Dimetrodon. Thus, they missed the direct link to the answer and resorted to another tool. In our previous research, we have found that users often have trouble utilizing information from graphics (Instone, Brown, Leventhal, Teasley, 1993); this result indicates that information in graphics is often overlooked and users have difficulty using tools that are embedded in graphics. For the Brachiosaurus question, the link in the text was not marked in any way. Even though the experimenter demonstrated the Hypertext method, no one actually used it.

For the two questions that were best answered with the Geographic Tool (Kansas and Alaska), $52.9 \%$ and $64.7 \%$, respectively, used only the globe tool to answer the question. For these questions, approximately one-third of the users
(34.9\% for Kansas and $29.4 \%$ for Alaska) chose the globe in combination with other tools.

\subsection{Accuracy}

Each answer was scored for accuracy using a binary scale. A correct answer was scored as 1 and both an incorrect answer and a "don't know" was scored as 0 . The average percent accuracy per question is given in Table 2.

Accuracy scores were analyzed using a withinsubjects analysis of variance (ANOVA). The main effect of question type was significant $(F(7,120)=$ $4.81, \mathrm{p}<.0001)$. The three questions answered most accurately were three of the one-fact questions: Alaska, Pteranodon and Allosaurus. The Kansas question was also answered accurately. These four questions require only one fact to answer the question. In addition, both of the questions that were best answered with the Index Tool are included among these three questions. This result suggests that users generally were able to get to single specified facts, especially when those facts were easy to find with the index.

The two least-accurately answered questions were Brachiosaurus and Height. For the Height question, most users answered it incorrectly because they used the default setting of the slide bar (weight), instead of height. Changing the slide bar mode to height required an extra operation on an unlabeled down-arrow button. This points out yet again the difficulty that users have in switching modes and being aware of modes.

\subsection{Speed}

The time to answer each question (in seconds) was recorded from the moment when the user started answering a question until an answer was given. The results are shown in Table 2. Speed scores were analyzed using a within-subject analysis of variance (ANOVA). There was a significant main effect of question type $(F(7,120)$ $=5.13, \mathrm{p}<.0001)$. The questions answered the fastest were Mammal (Category), Height (Slide Bar) and Allosaurus (Index). Allosaurus was 
answered quickly, accurately, and with the appropriate tool, suggesting that subjects were able to use the Index method effectively. Height was answered quickly but with poor accuracy, suggesting that while the overall function of the slide bar was evident, the necessity to change its mode was not.

Kansas and Brachiosaurus took the longest time to answer. Brachiosaurus was best answered with a hypertext link; obviously, because subjects did not use the link, they required more time to use another tool. Kansas required use of the Geographic Tool. In a follow-up questionnaire, seven of the users mentioned that the tool was too slow. For example, user number 17 wrote, "It was kind of confusing, especially trying to move the map" and user 13 wrote, "On the map when you clicked to go a direction it took a while for the screen to change." Of the $90 \%$ of the users who accessed the Geographic Tool for these two questions, about half used only the globe, while the other half used additional tools. Apparently when users chose the globe, the slow performance of the tool led them to chose an alternative.

\section{DISCUSSION AND CONCLUSIONS}

The primary purpose of the study was to explore the effectiveness of non-keyboard based searching in a realistic, kiosk-type, multimedia system. The most important and salient result is that users were most likely to chose the correct tool, answer accurately, and answer quickly for a question that was appropriately answered with the Index Tool. This pattern reflects, we believe, a familiarity and comfort with a text-based, hierarchicallyorganized style of information retrieval. The users had all been taught to use the school's card catalog, and probably found the library index a helpful metaphor. Naturally, the effectiveness of this tool was enhanced by the effective organization of the index contents. An open question for designers of search indexes for kiosk systems is how to structure an index, in terms of depth versus breadth. There is no doubt a tradeoff between a deep hierarchy (i.e., with subheadings and sub-subheadings) and a broader (longer) index (with no subheadings). While many studies have considered this question for menu structures (e.g., Landauer \& Nachbar, 1985), it is clearly an issue which needs to be dealt with in designing an index for keyboardless searching.

Because the Geographic Tool was slow, it apparently led some users to confirm their answers with, or switch to, another tool. Thus, our results point out that response time can effect usability, and, interestingly, users' acceptance of the validity of the information. Users did not use the hypertext capabilities at all, pointing out that an invisible tool or a tool that they do not notice because it is embedded in graphics will not get used, at least under the training conditions of this study. Also, users had trouble with the three modes assigned to the one slide bar; by having instead three slide bars and eliminating the modes, one would expect better performance by users.

This results of this study are supportive of the viability of keyboardless searching techniques in kiosk-style systems with non-trivial databases. However, the study does leave several questions unanswered. First, the users here were given a brief demonstration of the system. In a true kiosk situation, such training would not be givenwithout training, would users discover nontraditional tools like the Geographic Tool? A second question is whether adults or senior citizens would find the system as usable as our child users. Again, a true kiosk might be in a public situation and open to users of quite varied demographic characteristics. Finally, would alternative input devices, such as touch screens, speech, or other pointing devices effect usability, and if so, how?

\section{REFERENCES}

Heller, R. S. (1991) Computing Access in Public Spaces: Design Lessons Learned. Human Aspects in Computing: Design and Use of Interactive Systems and Work with Terminals., 699-703.

Hill, A. (1993) Dinosaur Adventure - User Guide, Knowledge Adventure.

Instone, K., Brown, E., Leventhal, L., Teasley, B. (1993) The Challenge of Effectively Integrating Graphics into Hypertext. In L. Bass, J. Gornostaev \& C. Unger (Eds.), HumanComputer Interaction: EWHCI '93 Selected Papers, Berlin: Springer-Verlag, 290-297.

Landauer, T. \& Nachbar, D. Selection from Alphabetic and Numeric Menu Trees Using a Touch Screen. CHI '85 Proceedings, 73-78.

Leventhal, L., Instone, K. \& Teasley, B. (1994) Hypertext-based Kiosk Systems: Seven Challenges and an Empirical Study. In B. Blumenthal, et al. (Eds.), Human-Computer Interaction: EWHCI ' 94 Selected Papers, Berlin: Springer-Verlag, 226-237.

Salomon, G. (1990) Designing Casual-Use Hypertext: The CHI ' 89 InfoBooth, CHI 90 Proceedings, 451-458. 